\title{
Intraperitoneal Infusion of Mesenchymal Stem/Stromal Cells Prevents Experimental Autoimmune Uveitis in Mice
}

\author{
Joo Youn Oh, ${ }^{1,2}$ Tae Wan Kim, ${ }^{3}$ Hyun Jeong Jeong, ${ }^{2}$ Hyun Ju Lee, ${ }^{2}$ \\ Jin Suk Ryu, ${ }^{2}$ Won Ryang Wee, ${ }^{1,2}$ Jang Won Heo, ${ }^{1}$ and Mee Kum Kim ${ }^{1,2,4}$ \\ ${ }^{1}$ Department of Ophthalmology, Seoul National University Hospital, Seoul 110-744, Republic of Korea \\ ${ }^{2}$ Laboratory of Ocular Regenerative Medicine and Immunology, Seoul Artificial Eye Center, \\ Seoul National University Hospital Biomedical Research Institute, Seoul 110-744, Republic of Korea \\ ${ }^{3}$ Department of Ophthalmology, Seoul National University Boramae Medical Center, Seoul 156-707, Republic of Korea \\ ${ }^{4}$ Department of Ophthalmology, Seoul National University College of Medicine, 103 Daehak-ro, Jongno-gu, \\ Seoul 110-799, Republic of Korea \\ Correspondence should be addressed to Mee Kum Kim; kmk9@snu.ac.kr
}

Received 6 March 2014; Revised 23 June 2014; Accepted 25 June 2014; Published 17 July 2014

Academic Editor: Francisco J. Ascaso

Copyright (C) 2014 Joo Youn Oh et al. This is an open access article distributed under the Creative Commons Attribution License, which permits unrestricted use, distribution, and reproduction in any medium, provided the original work is properly cited.

\begin{abstract}
Autoimmune uveitis is one of the leading causes of blindness. We here investigated whether intraperitoneal administration of human mesenchymal stem/stromal cells (hMSCs) might prevent development of experimental autoimmune uveitis (EAU) in mice. Time course study showed that the number of IFN- $\gamma$ - or IL-17-expressing CD $4^{+} \mathrm{T}$ cells was increased in draining lymph nodes (DLNs) on the postimmunization day 7 and decreased thereafter. The retinal structure was severely disrupted on day 21. An intraperitoneal injection of hMSCs at the time of immunization protected the retina from damage and suppressed the levels of proinflammatory cytokines in the eye. Analysis of DLNs on day 7 showed that hMSCs decreased the number of Th1 and Th17 cells. The hMSCs did not reduce the levels of IL-1 $\beta$, IL-6, IL-12, and IL-23 which are the cytokines that drive Th1/Th17 differentiation. Also, hMSCs did not induce $\mathrm{CD} 4{ }^{+} \mathrm{CD} 25^{+}$Foxp $3^{+}$cells. However, hMSCs increased the level of an immunoregulatory cytokine IL-10 and the population of IL-10-expressing $\mathrm{B} 220^{+} \mathrm{CD} 19^{+}$cells. Together, data demonstrate that hMSCs attenuate EAU by suppressing Th1/Th17 cells and induce IL-10-expressing B220 ${ }^{+} \mathrm{CD} 19^{+}$cells. Our results support suggestions that hMSCs may offer a therapy for autoimmune diseases mediated by Th1/Th17 responses.
\end{abstract}

\section{Introduction}

Autoimmune uveitis of noninfectious origin is a visionthreatening disease that affects 115.3 per 100,000 people and accounts for $2.8-10 \%$ of all cases of blindness in the United States [1]. Corticosteroids are the first line of therapy for patients with autoimmune uveitis. However, long-term use of corticosteroids is associated with serious systemic and ocular adverse effects. Also, there are subtypes of autoimmune uveitis that are refractory to steroids [2]. For these reasons, efforts are being made to develop new therapies for autoimmune uveitis by modulating immune responses underlying the pathogenesis of uveitis. The pathogenesis of autoimmune uveitis is traditionally regarded as Thl-mediated $[3,4]$. However, Th17 cell is recently identified as a novel subset of $\mathrm{T}$ cells that contributes to the development of autoimmune diseases including uveitis $[3,4]$.

Stromal progenitors of mesodermal cells, referred to as mesenchymal stem cells or multipotent mesenchymal stromal cells (MSCs), have the remarkable capacity to protect tissues from various immune-mediated diseases. Studies demonstrate that MSCs exert immunosuppressive functions by modulating Th1, Th2, or Th17 immune responses, by inhibiting $\mathrm{T}$ cell proliferation, or by generating regulatory $\mathrm{T}$ (Treg) cells [5-7]. Also, clinical trials are in progress to capitalize on the immunomodulatory effects of MSCs for treatment of patients with autoimmune diseases such as multiple sclerosis, Crohn's disease, type 1 diabetes, systemic lupus erythematous, or systemic sclerosis [8]. 
In this study, we investigated whether an intraperitoneal (IP) administration of human bone marrow-derived MSCs (hMSCs) might prevent development of experimental autoimmune uveitis (EAU) in mice, a model for human autoimmune uveitis.

\section{Materials and Methods}

2.1. Animals. Six-week-old female B6 mice (C57BL/6J) were purchased from Orient Bio Inc. (Seongnam, Korea) and maintained in a specific pathogen-free environment with continuously available water and food. Animals were treated in strict accordance with the ARVO statement for the use of animals in ophthalmic and vision research. The experimental protocols were approved by the Institutional Animal Care and Use Committee of Seoul National University Biomedical Research Institute.

2.2. Preparation of hMSCs. Human bone marrow-derived MSCs were obtained from the Center for the Preparation and Distribution of Adult Stem Cells (http://medicine.tamhsc .edu/irm/msc-distribution.html) that supplies standardized preparations of MSCs enriched for early progenitor cells to over 300 laboratories under the auspices of an NIH/NCRR grant (P40 RR 17447-06). Animal experiments were performed with passage two hMSCs from one donor. The cells consistently differentiated into three lineages in culture, were negative for hematopoietic markers (CD34, CD36, CD117, and CD45), and were positive for mesenchymal markers CD29 (95\%), CD44 (>93\%), CD49c (99\%), CD49f (>70\%), CD59 (>99\%), CD90 (>99\%), CD105 (>99\%), and CD166 (>99\%). The cells were cultured in complete culture medium with $16 \%$ FBS until $70 \%$ confluence was reached and harvested with $0.25 \%$ trypsin $/ 1 \mathrm{mM}$ EDTA at $37^{\circ} \mathrm{C}$ for $2 \mathrm{~min}$. After washing, the cells were resuspended in balanced salt solution (BSS; BioWhittaker, Walkersville, MD) at a concentration of 10,000 cells $/ \mu \mathrm{L}$ for injection in vivo.

2.3. Induction and Treatment of EAU. EAU was induced in mice by subcutaneous injection into a footpad of $250 \mu \mathrm{g}$ human interphotoreceptor retinoid binding protein (IRBP) peptide 1-20, GPTHLFQPSLVLDMAKVLLD $(20 \mathrm{mg} / \mathrm{mL}$; Peptron, Daejeon, Korea), that was emulsified in complete Freund adjuvant (Sigma, Saint Louis, MO) containing Mycobacterium tuberculosis $(2.5 \mathrm{mg} / \mathrm{mL}$; BD Difco, Franklin Lakes, NJ). Simultaneously, the mice received $0.7 \mu \mathrm{g}$ Pertussis toxin $(300 \mu \mathrm{L}$; Sigma) intraperitoneally. Immediately after immunization, either $1 \times 10^{6} \mathrm{hMSCs}$ in $100 \mu \mathrm{L}$ BSS or BSS $(100 \mu \mathrm{L})$ alone was injected intraperitoneally.

2.4. Histology and Histological Scoring. On days 7, 14, and 21 after immunization, mice were humanely killed, and eyeballs, inguinal and popliteal LNs were collected for further assays. Eyeballs were fixed in $10 \%$ formaldehyde and paraffin-embedded. Serial $4 \mu \mathrm{m}$ thick sections were cut and stained with either hematoxylin/eosin or TUNEL (terminal deoxynucleotidyl transferase dUTP nick end labeling) as the manufacturer's protocol (Chemicon International, Temecula,
CA). The morphologic features of the retina were examined, and histological disease scores were assessed by two independent observes (Joo Youn Oh and Tae Wan Kim) in a blinded manner on a scale of 0 to 4 using the criteria previously defined by Caspi [9].

2.5. Flow Cytometric Analysis. The proportions of Th1, Th17, $\gamma \delta \mathrm{T}$, and Treg cells were determined by measuring IFN- $\gamma$, IL-17, $\gamma \delta$ TCR, or CD25 and Foxp3-expressing CD $4^{+}$cells using flow cytometry. In addition, the population of IL-10 expressing cells was determined by staining the cells with CD4, CD25, Foxp3, CD19, B220, CD11b, CD11c, and IL10. To collect cell suspensions, popliteal or inguinal nodes (LNs) were placed and minced between the frosted ends of two glass slides in RPMI media containing 10\% FBS and $1 \%$ penicillin-streptomycin. The cells were immunostained with the following fluorescence-conjugated anti-mouse antibodies: CD4, CD25, Foxp3, IFN- $\gamma, \gamma \delta$ TCR, CD19, B220, CD11b, CD11c, IL-10 (eBioscience, San Diego, CA), and IL17A (BD Pharmingen, San Diego, CA). For intracellular staining, the cells were stimulated for $4 \mathrm{~h}$ with $50 \mathrm{ng} / \mathrm{mL}$ phorbol myristate acetate and $1 \mu \mathrm{g} / \mathrm{mL}$ ionomycin in the presence of GolgiPlug (BD Pharmingen). The cells were then assayed for fluorescence using a FACSCanto flow cytometer (BD BioSciences, Mountain View, CA). The gate was set on $\mathrm{CD}^{+}$or $\mathrm{CD} 19^{+}$cell population, and further analysis of surface or intracellular markers was done within this gate. Data were analyzed using Flowjo program (Tree Star, Inc., Ashland, OR).

2.6. Real-Time RT-PCR. LNs were lysed in RNA isolation reagent (RNA Bee, Tel-Test Inc., Friendswood, TX) and homogenized using a sonicator (Ultrasonic Processor, Cole Parmer Instruments, Vernon Hills, IL). Total RNA was extracted using RNeasy Mini kit (Qiagen, Valencia, CA) and used to synthesize double-stranded cDNA by reverse transcription (SuperScript III, Invitrogen, Carlsbad, CA). Real-time amplification was performed using TaqMan Universal PCR Master Mix (Applied Biosystems, Carlsbad, CA). An $18 \mathrm{~s}$ rRNA probe (TaqMan Gene Expression Assays ID, Hs03003631_g1) was used for normalization of gene expression. For all the PCR probe sets, TaqMan Gene Expression Assay kits were purchased from Applied Biosystems: IL-1 $\beta$ (Mm00434228_m1), IL-6 (Mm00446190_m1), IFN- $\gamma$ (Mm01168134_m1) IL-17A (Mm00439618_m1), IL10 (Mm00439614_ml), TGF- $\beta$ (Mm01178820_m1), IL-12A (Mm00434165_ml), and IL-23 (Mm01160011_g1), and human GAPDH (Hs02758991_g1).

2.7. ELISA. For protein extraction, eyeballs were cut into small pieces and lysed in PRO-PREP Protein Extraction Solution (Intron Biotechnology, Seongnam, Korea). The samples were sonicated on ice using an ultrasound sonicator. After centrifugation at $12,000 \mathrm{rpm}$ for $20 \mathrm{~min}$, the supernatant was collected and assayed for IFN- $\gamma$ by ELISA according to the manufacturer's protocol (DuoSet; R \& D Systems, Minneapolis, MN). 
2.8. Statistical Analysis. Values were compared between the groups using the one-way ANOVA (SPSS 12.0, Chicago, IL) or Student's $t$ test and shown as the mean value \pm standard error (SEM). Differences were considered significant at $P<0.05$.

\section{Results}

3.1. hMSCs Reduced Retinal Damage in Mice with EAU. EAU was induced in mice by subcutaneous injection of IRBP in a footpad on day 0 . Simultaneously, either hMSCs $\left(1 \times 10^{6}\right.$ cells/mouse) or BSS was injected intraperitoneally. On days 7, 14 , and 21 , the mice were humanely killed, and eyeballs and draining LNs (DLNs) were collected for assays (Figure 1(a)). ELISA showed that the level of the proinflammatory cytokine IFN- $\gamma$ was markedly increased in the eyeball on day 14 and significantly reduced by treatment with hMSCs (Figure 1(b)). Histology demonstrated that the retinal structure including the photoreceptor layer was severely disorganized with massive infiltration of inflammatory cells in the vitreous cavity and in the retina of BSS-treated EAU mice on day 21 (histological score $2.13 \pm 0.31$, Figures $1(\mathrm{c})$ and $1(\mathrm{~d})$ ). In contrast, the retinal architecture was almost completely preserved with few inflammatory cells in hMSCs-treated mice on day 21 (histological score $0.25 \pm 0.14$, Figures 1 (c) and $1(\mathrm{~d})$ ). Similarly, TUNEL staining indicated the presence of many dead cells in the photoreceptor layer in BSS-treated EAU mice on day 21, while there were few TUNEL-positive cells in mice treated with hMSCs (Figure 1(e)).

To determine whether hMSCs suppressed the intraocular inflammation by direct contact, we evaluated the presence of hMSCs in the eye by real-time RT-PCR assays for humanspecific GAPDH. However, we did not detect any amplification of human GAPDH, indicating that hMSCs were not present in the eye on days 7, 14, or 21 after IP injection.

Therefore, the data clearly indicated that a single IP injection of hMSCs at the time of immunization prevented the development of EAU and protected the retina from inflammation-mediated damage.

3.2. hMSCs Decreased Th1/Th17 Cells in DLNs. We next investigated whether the tissue-protective effects of hMSCs might be due to the influence of hMSCs on the development of Th1, Th17, or $\gamma \delta \mathrm{T}$ cells that are pathogenic effectors in uveitis $[3,4]$. Time course study showed that the percentage of IFN- $\gamma$-expressing $\mathrm{CD} 4^{+}$cells, IL-17A-expressing $\mathrm{CD} 4^{+}$ cells, and $\gamma \delta$ TCR-expressing $\mathrm{CD}^{+}$cells was significantly increased in popliteal and inguinal LNs of EAU mice on day 7 and decreased thereafter to baseline levels (Figure 2, Supplemental Figure 1 (see Supplemental Figure 1 available online at http://dx.doi.org/10.1155/2014/624640)). Treatment with hMSCs significantly reduced the percentage of Th1 and Th17 cells in popliteal and inguinal LNs on day 7 (Figure 2). Consistently, the levels of IL-17A and IFN- $\gamma$ transcripts were significantly reduced by hMSCs, compared to the BSS-treated controls (Figure 3(a)). However, the percentage of $\gamma \delta \mathrm{T}$ cells was not affected by hMSC treatment (Supplemental Figure 1).

In order to determine whether hMSCs inhibited Th1 or Th17 cell generation by suppressing the production of
Th1- or Th17-polarizing cytokines, we further measured the levels of IL-1 $\beta$, IL- 6 , IL-12a, IL-23, and TGF- $\beta$ which are the cytokines that drive the development of Th1 and Th17 cells [10-12]. However, hMSCs did not reduce the levels of IL$1 \beta$, IL-6, IL-12A, IL-23, and TGF- $\beta$ in DLNs (Figures 3(b)$3(d))$. Of note, hMSCs significantly increased the level of IL10, an immunoregulatory cytokine that suppresses Th1/Th17 immune responses (Figure 3(b)).

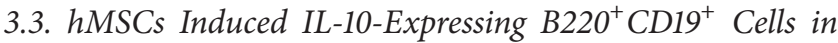
DLNs. To identify the IL-10-expressing cell population, we examined $\mathrm{CD}^{+}, \mathrm{CD}^{+} 9^{+}, \mathrm{B} 220^{+}, \mathrm{CD}_{11 b^{+}}$, or $\mathrm{CD} 11 \mathrm{c}^{+}$cells in DLNs for IL-10 expression. We found that the percentage of $\mathrm{IL}-10^{+} \mathrm{B} 220^{+} \mathrm{CD} 19^{+}$cells was markedly increased in popliteal LNs of hMSCs-treated EAU mice on day 7, compared to BSS-treated EAU mice (Figures 4(a) and 4(b)). However, IL-10 expression was not increased in $\mathrm{CD}^{+}, \mathrm{CD}^{+} \mathrm{b}^{+}$, or $\mathrm{CD}_{11 c^{+}}$cells (data not shown). Also, hMSCs did not increase the percentage of $\mathrm{CD} 4^{+} \mathrm{CD} 25^{+}$Foxp $3^{+}$Treg cells in popliteal or inguinal LNs at all examined time-points (Supplemental Figures 2(a) and 2(b)). Time course indicated that $\mathrm{CD} 4^{+} \mathrm{CD} 25^{+}$Foxp $3^{+}$cells initially increased in popliteal and inguinal LNs on day 7 after EAU induction and gradually decreased to baseline on days 14 and 21 . On the contrary, $\mathrm{CD} 4^{+} \mathrm{CD} 25^{+}$Foxp $^{+}$cells initially decreased in the spleen on day 7 and increased thereafter until day 21 . The hMSCs significantly suppressed an increase of $\mathrm{CD} 4^{+} \mathrm{CD} 25^{+}$Foxp $3^{+}$cells in the spleen on days 14 and 21 (Supplemental Figure 2(c)), reflecting that hMSCs might suppress early inflammation that is required for initial Treg expansion [11].

\section{Discussion}

Time course study revealed that Th1 and Th17 cells increased in DLNs after EAU immunization, and then the levels of proinflammatory cytokines markedly increased in the eyes. Subsequently, the retinal structure was severely and irreversibly disrupted. A single IP administration of hMSCs at the time of immunization significantly decreased Th1 or Th17 cells in DLNs and increased $\mathrm{IL}_{10}{ }^{+} \mathrm{B} 220^{+} \mathrm{CD} 19^{+}$cells on day 7. As a result, ocular inflammation was markedly repressed, and the retina was almost completely protected from damage. Therefore, our data suggest that hMSCs ameliorate autoimmune uveitis by suppressing the Th1/Th17 immune responses.

There are several possibilities that account for the mechanisms of hMSCs in repressing the Th1/Th17 immune responses. One is that hMSCs may directly inhibit differentiation or expansion of Th1/Th17 cells. In support of this hypothesis, previous reports demonstrated that MSCs suppressed T cell proliferation and inhibited differentiation of naïve $\mathrm{CD} 4^{+}$ T cells into Th1 or Th17 cells [13-18]. However, it is unclear in vivo whether enough number of systemically administered hMSCs reaches peripheral LNs or injured tissues to exert direct inhibitory effects on T cells.

Another possibility is that hMSCs may modulate host cells to suppress immune responses. This is likely because a single administration of MSCs that are short-lived has long-term effects in the host as shown in our study. In fact, 


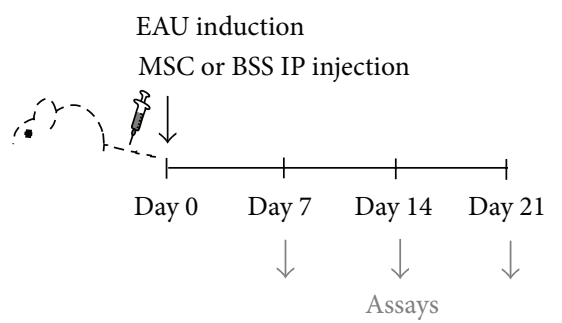

(a)

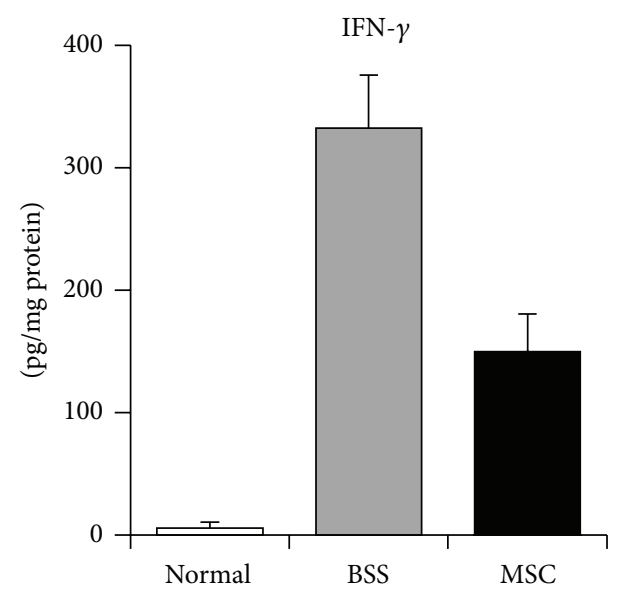

(b)
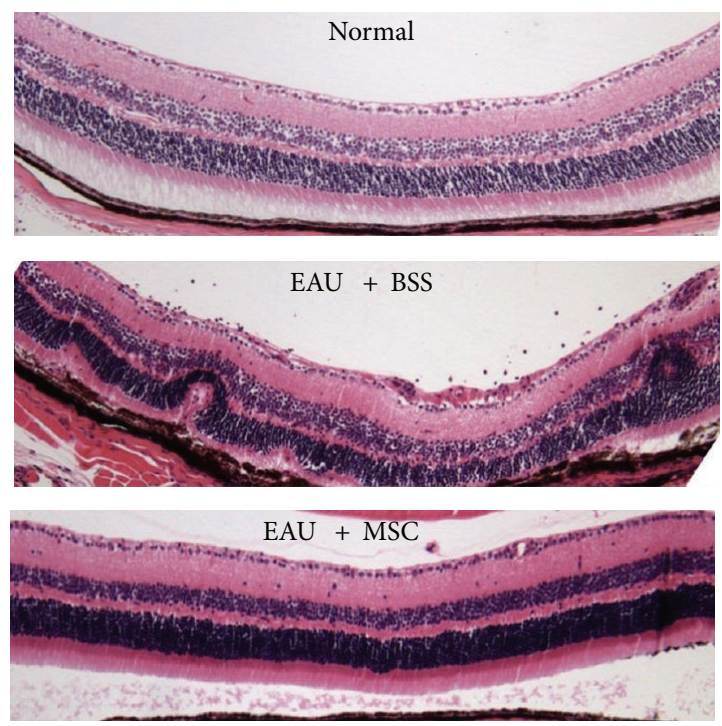

(d)

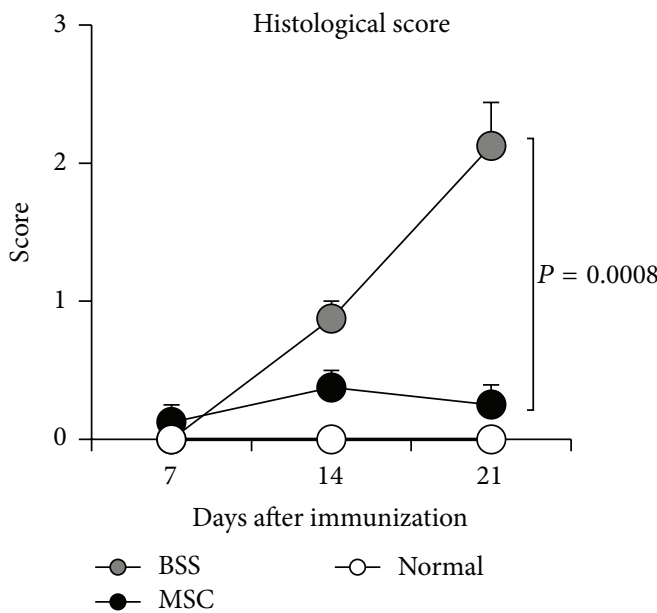

(c)
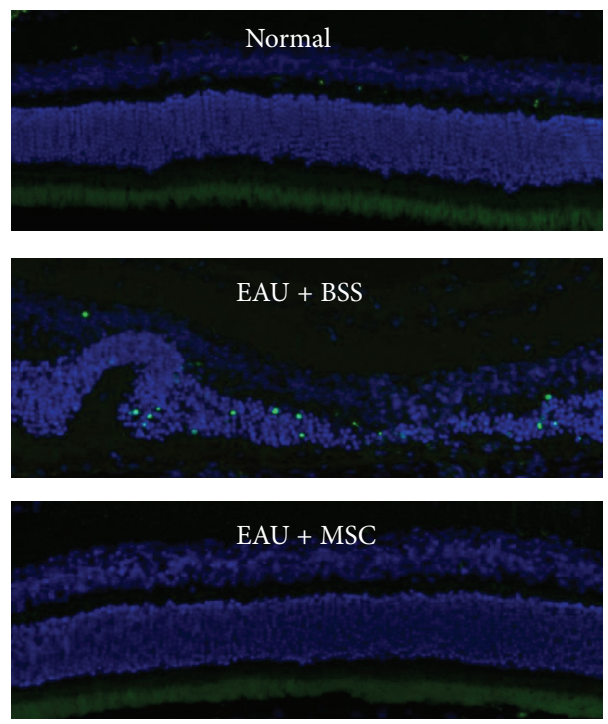

(e)

FIgURE 1: Histological findings of the eye. (a) EAU was induced in mice on day 0 , and either hMSCs $\left(1 \times 10^{6}\right.$ cells in $100 \mu \mathrm{L}$ BSS $)$ or BSS $(100 \mu \mathrm{L})$ were intraperitoneally (IP) injected immediately after EAU induction. On days 7, 14, and 21, the eyes or DLNs were collected for assays. (b) ELISA showed that IFN- $\gamma$ in the eye was markedly increased in the eyeball on day 14 and significantly reduced by hMSCs. Data are presented in mean + SEM. $n=5$ in each group. (c) Time course of histological disease scores demonstrated that the retinal pathology gradually developed with a peak at day 21 . Histological scores were significantly lower in hMSCs-treated mice at all time-points, suggesting that hMSCs prevented the disease development. Data are presented in mean + SEM. $n=5$ in each group. (d) Hematoxylin-eosin staining of the eye on day 21 showed severe disruption of the retinal structure including the photoreceptor layer with inflammatory cell infiltration in the vitreous cavity and in the retina of EAU mice. In contrast, the retinal structure was well-reserved, and few inflammatory cells were observed in EAU mice treated with hMSCs. (e) TUENL staining showed a number of dead cells in the disrupted photoreceptor layer of EAU mice. In contrast, no TUENL-positive cells were found in the retina of mice treated with hMSCs. 

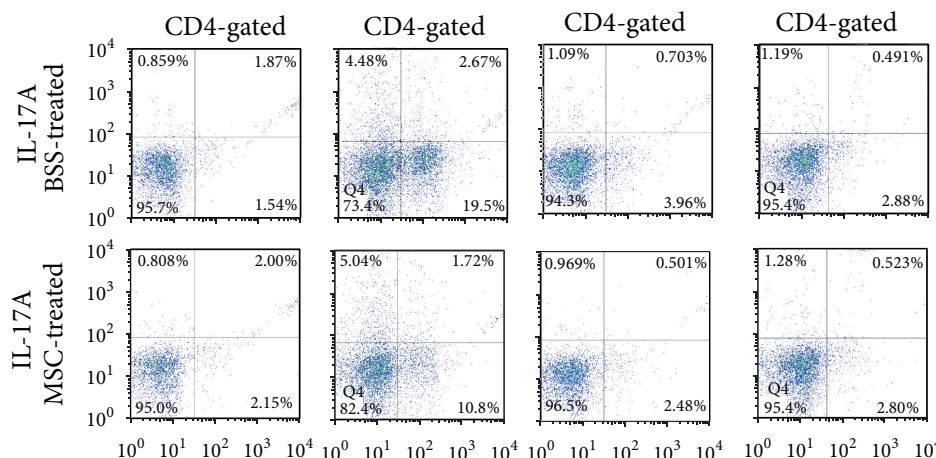

$$
\begin{array}{llll}
\text { Day 0 } & \text { Day 7 } & \text { Day 14 } & \text { Day 21 } \\
\text { IFN- } \gamma & \text { IFN- } \gamma & \text { IFN- } \gamma & \text { IFN- } \gamma
\end{array}
$$

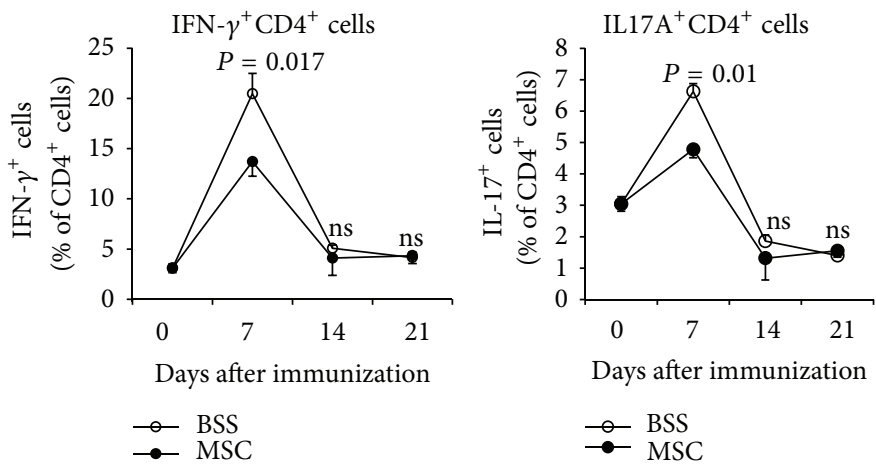

(a)
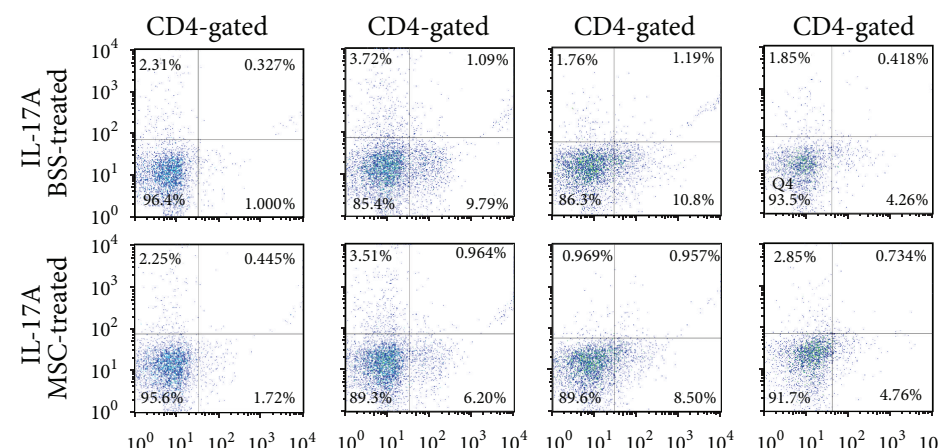

Day 0

$$
\begin{aligned}
& \text { Day } 7 \\
& \text { IFN- } \gamma
\end{aligned}
$$

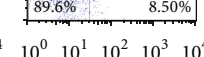

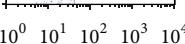

IFN- $\gamma$

$$
\text { Day } 14
$$

Day 21

IFN- $\gamma$

IFN- $\gamma$
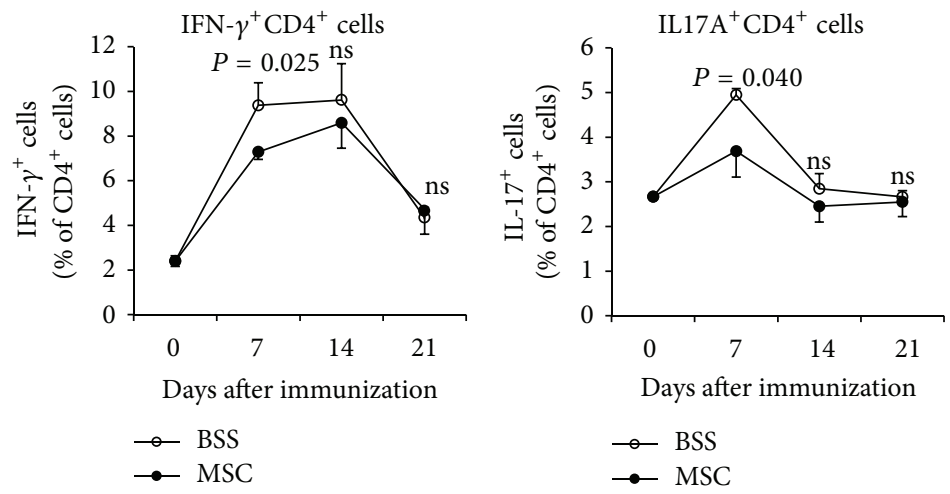

(b)

FIgURE 2: Flow cytometry for Th1 and Th17 cells in LNs. Time course study demonstrated that the percentages of IFN- $\gamma$-expressing CD4 ${ }^{+}$ cells or IL-17A-expressing $\mathrm{CD} 4^{+}$cells were significantly increased in popliteal (a) and inguinal LNs (b) on day 7 after EAU induction and decreased thereafter to baseline until day 21. The percentages of both Th1 and Th17 cells were significantly lower in EAU mice treated with hMSCs, compared to BSS-treated mice. Data are presented in mean \pm SEM. $n=5$ in each group. 


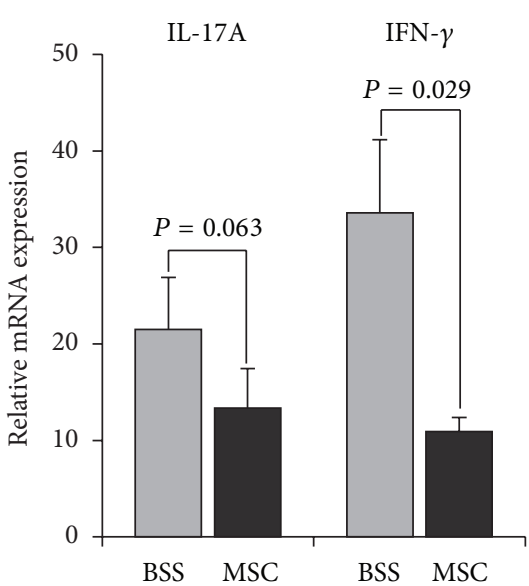

(a)

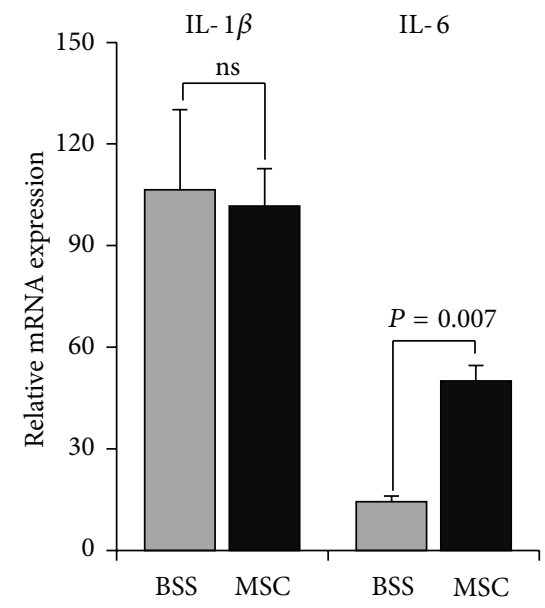

(c)

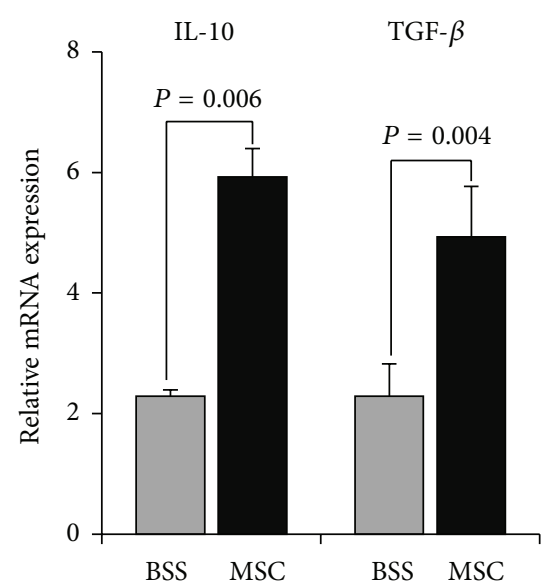

(b)

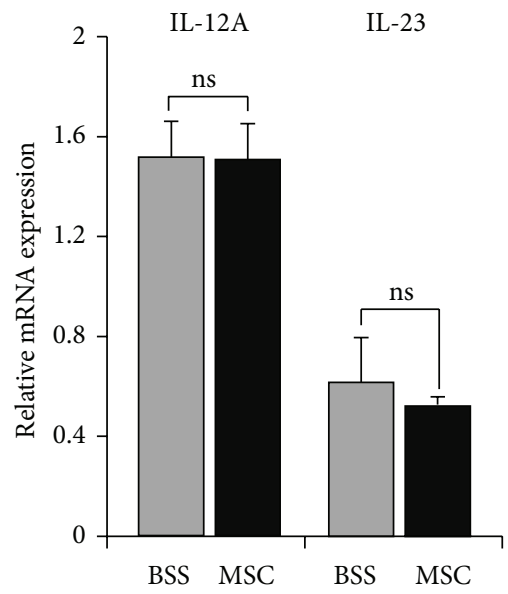

(d)

FIGURE 3: Assay for inflammation- and immune-related cytokines in LNs. Real-time RT-PCR analysis showed that the levels of IL-17A and IFN- $\gamma$ transcripts were increased in popliteal LNs on day 7 after EAU induction and significantly reduced by hMSCs (a). However, the levels of IL-10, TGF- $\beta$, and IL- 6 were significantly increased by hMSCs (b, c). The levels of IL-1 $\beta$, IL-12A, or IL-23 were not different between BSSand hMSC-treated EAU mice (c, d). Data are presented in mean + SEM. $n=5$ in each group.

a number of previous studies showed that MSCs induced Treg cells $[18,19]$, switched microglia or macrophages toward anti-inflammatory or tissue-repairing phenotypes [20-22], or promoted generation of regulatory or tolerogenic dendritic cells [23-25]. Consistent with these reports, we found that the level of IL-10, an immunoregulatory cytokine that suppresses Th1/Th17 immune responses [26, 27], was markedly upregulated in DLNS of mice treated with hMSCs. Among the examined cell populations $\left(\mathrm{CD} 4^{+}, \mathrm{CD}_{1} 9^{+}, \mathrm{CD}_{11 \mathrm{~b}}{ }^{+}\right.$, and

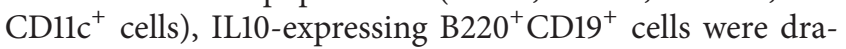
matically increased. IL-10-producing $B$ cells are known to be critical for suppression of autoimmune diseases, although $\mathrm{B}$ cells are both pathogenic and protective in autoimmune diseases [28]. Given that the effects of MSCs on B cells have been rarely studied so far, further characterization of IL10expressing $\mathrm{B} 220^{+} \mathrm{CD} 19^{+}$cells as a potential regulatory B cell subset $[29,30]$ would provide a mechanistic insight into the immunomodulatory mechanism of hMSCs.
Since the therapeutic efficacy of MSCs was first reported in experimental autoimmune encephalitis [31], there have been efforts to use MSCs for treating a variety of autoimmune diseases such as autoimmune arthritis, autoimmune myocarditis, or Sjögren's syndrome $[5,8,16,17,19]$. When it comes to autoimmune uveitis, two studies recently demonstrated that MSCs attenuated EAU in mice and rats. Tasso et al. [32] reported that a single IP injection of syngeneic mouse MSCs at the time of immunization almost completely reduced the incidence and severity of disease in mice with EAU induced by IRBP injection. They found that the percentage of $\mathrm{CD} 4^{+} \mathrm{CD} 25^{+}$Foxp $3^{+}$cells was significantly higher in the spleen in MSC-treated EAU mice than in untreated controls. Another study by Zhang et al. [33] reported that an intravenous administration of syngeneic or allogeneic rat MSCs strikingly reduced the severity of EAU induced by IRBP in rats. MSCs were effective when the cells were administered before the onset or at the peak of disease, but 


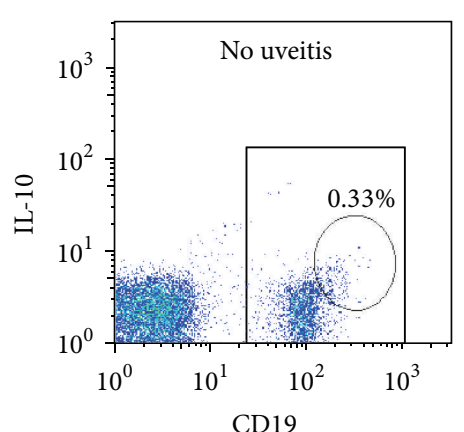

CD19
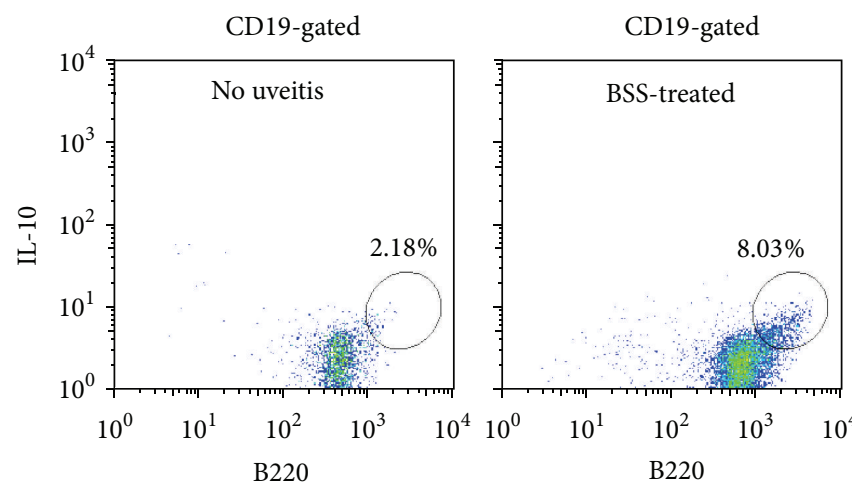

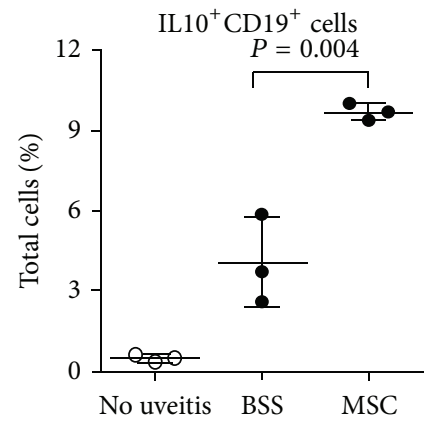

CD19
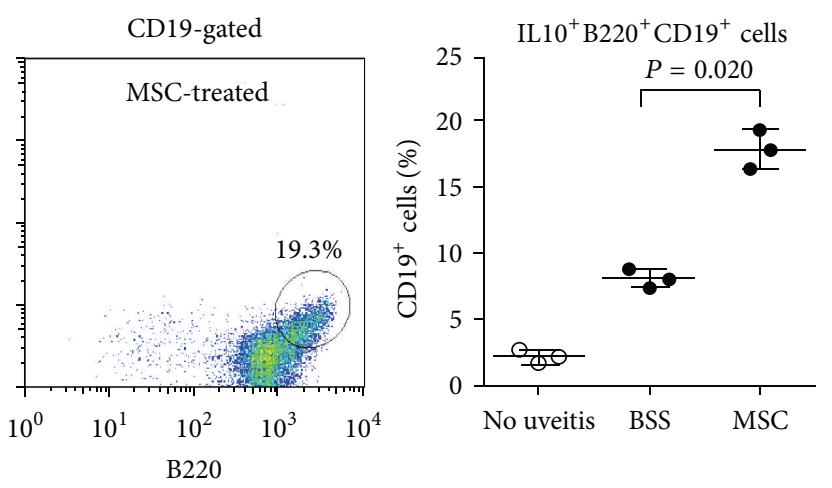

(b)

FIGURE 4: Assay for IL-10-expressing cells in LNs. Flow cytometric analysis of popliteal LNs on day 7 after EAU immunization showed that the percentages of IL- $10^{+} \mathrm{CD} 19^{+}$(a) and IL- $10^{+} \mathrm{B} 220^{+} \mathrm{CD} 19^{+}$cells (b) were significantly increased in mice treated with hMSCs, compared to mice without EAU or BSS-treated EAU mice. Data are presented in mean \pm SEM. $n=5$ in each group.

not after disease stabilization. They additionally found that the levels of IL-2, IL-17, and IFN- $\gamma$ were lower in supernatants of T lymphocytes isolated from EAU mice treated with MSCs, compared to $\mathrm{T}$ lymphocytes from untreated EAU mice. However, either study did not directly evaluate the effects of MSCs on Th1 and Th17 cells in DLNs in vivo as in our study.

Another difference of our study is that we used human MSCs for the study. Mouse MSCs undergo spontaneous transformation during expansion in culture and occasionally become tumorigenic in the same manner as mouse fibroblasts [34-36]. Moreover, some of the immunomodulatory effects of MSCs are species-specific. For instance, indolamine 2, 3dioxygenase (IDO) is involved in the immunosuppressive activity of human MSCs, whereas inducible nitric oxide synthase (iNOS) mediated the immunosuppressive activity of mouse MSCs [37]. Therefore we used hMSCs for this study in order to evaluate clinical efficacy of hMSCs and their mechanism(s) as a potential treatment of autoimmune uveitis in humans. As a result, we found that the number of Th1 and Th17 cells was markedly reduced by hMSCs and $\mathrm{IL}_{10}{ }^{+} \mathrm{B} 220^{+} \mathrm{CD} 19^{+}$cells, not classical $\mathrm{CD} 4^{+} \mathrm{CD} 25^{+} \mathrm{Foxp}^{+}$ Treg cells, were increased by hMSCs. Further studies using a specific depletion of IL-10 production from B cells would be necessary to determine whether IL-10-producing B cells are a main mediator of hMSCs in suppressing EAU.

\section{Conclusions}

In conclusion, our data demonstrate that systemic administration of hMSCs almost completely prevented the development of EAU by suppressing Th1/Th17 immune responses and protected the retina from immune-mediated damage. The results provide a further rationale for the use of hMSCs to treat a variety of autoimmune or immune-mediated diseases involving the eye and other organs that are driven by excessive Th1/Th17 immune responses.

\section{Conflict of Interests}

There is no conflict of interests to declare.

\section{Acknowledgments}

This work was supported by a grant of the Korean Health Technology R \& D Project, Ministry of Health \& Welfare, 
Republic of Korea (A112023), and the National Research Foundation of Korea Grant funded by the Korean Government (NRF 2011-0004128).

\section{References}

[1] D. C. Gritz and I. G. Wong, "Incidence and prevalence of uveitis in Northern California: the Northern California Epidemiology of Uveitis Study," Ophthalmology, vol. 111, no. 3, pp. 491-500, 2004.

[2] P. Lehoang, "The gold standard of noninfectious uveitis: corticosteroids," Developments in Ophthalmology, vol. 51, pp. 7-28, 2012.

[3] F. Willermain, J. T. Rosenbaum, B. Bodaghi et al., "Interplay between innate and adaptive immunity in the development of non-infectious uveitis," Progress in Retinal and Eye Research, vol. 31, no. 2, pp. 182-194, 2012.

[4] R. R. Caspi, "A look at autoimmunity and inflammation in the eye," Journal of Clinical Investigation, vol. 120, no. 9, pp. 30733083, 2010.

[5] A. Uccelli and D. J. Prockop, "Why should mesenchymal stem cells (MSCs) cure autoimmune diseases?" Current Opinion in Immunology, vol. 22, no. 6, pp. 768-774, 2010.

[6] A. Uccelli, L. Moretta, and V. Pistoia, "Mesenchymal stem cells in health and disease," Nature Reviews Immunology, vol. 8, no. 9, pp. 726-736, 2008.

[7] K. English, A. French, and K. J. Wood, "Mesenchymal stromal cells: facilitators of successful transplantation?" Cell Stem Cell, vol. 7, no. 4, pp. 431-442, 2010.

[8] A. Tyndall and A. Uccelli, "Multipotent mesenchymal stromal cells for autoimmune diseases: teaching new dogs old tricks," Bone Marrow Transplantation, vol. 43, no. 11, pp. 821-828, 2009.

[9] R. R. Caspi, "Experimental autoimmune uveoretinitis in the rat and mouse," Current Protocols in Immunology, vol. 15, pp. 1-12, 2003.

[10] A. O'Garra, "Cytokines induce the development of functionally heterogeneous T helper cell subsets," Immunity, vol. 8, no. 3, pp. 275-283, 1998.

[11] E. Bettelli, Y. Carrier, W. Gao et al., "Reciprocal developmental pathways for the generation of pathogenic effector TH17 and regulatory T cells," Nature, vol. 441, no. 7090, pp. 235-238, 2006.

[12] M. Veldhoen, R. J. Hocking, C. J. Atkins, R. M. Locksley, and B. Stockinger, "TGF $\beta$ in the context of an inflammatory cytokine milieu supports de novo differentiation of IL-17-producing $\mathrm{T}$ cells," Immunity, vol. 24, no. 2, pp. 179-189, 2006.

[13] M. Rafei, P. M. Campeau, A. Aguilar-Mahecha et al., "Mesenchymal stromal cells ameliorate experimental autoimmune encephalomyelitis by inhibiting CD4 Th17 T cells in a CC chemokine ligand 2-dependent manner," Journal of Immunology, vol. 182, no. 10, pp. 5994-6002, 2009.

[14] E. Svobodova, M. Krulova, A. Zajicova et al., "The role of mouse mesenchymal stem cells in differentiation of naive T-cells into anti-inflammatory regulatory $\mathrm{T}$-cell or proinflammatory helper T-cell 17 population," Stem Cells and Development, vol. 21, no. 6, pp. 901-910, 2012.

[15] X. Qu, X. Liu, K. Cheng, R. Yang, and R. C. H. Zhao, "Mesenchymal stem cells inhibit Th17 cell differentiation by IL10 secretion," Experimental Hematology, vol. 40, no. 9, pp. 761770, 2012.

[16] M. Ohshima, K. Yamahara, S. Ishikane et al., "Systemic transplantation of allogenic fetal membrane-derived mesenchymal stem cells suppresses Th1 and Th17 T cell responses in experimental autoimmune myocarditis," Journal of Molecular and Cellular Cardiology, vol. 53, no. 3, pp. 420-428, 2012.

[17] J. Xu, D. Wang, D. Liu et al., "Allogeneic mesenchymal stem cell treatment alleviates experimental and clinical Sjögren syndrome," Blood, vol. 120, no. 15, pp. 3142-3151, 2012.

[18] S. Ghannam, J. Pène, G. Torcy-Moquet, C. Jorgensen, and H. Yssel, "Mesenchymal stem cells inhibit human Th17 cell differentiation and function and induce a $\mathrm{T}$ regulatory cell phenotype," The Journal of Immunology, vol. 185, no. 1, pp. 302312, 2010.

[19] M. J. Park, H. S. Park, M. L. Cho et al., "Transforming growth factor $\beta$-transduced mesenchymal stem cells ameliorate experimental autoimmune arthritis through reciprocal regulation of Treg/Th17 cells and osteoclastogenesis," Arthritis and Rheumatism, vol. 63, no. 6, pp. 1668-1680, 2011.

[20] H. Ohtaki, J. H. Ylostalo, J. E. Foraker et al., "Stem/progenitor cells from bone marrow decrease neuronal death in global ischemia by modulation of inflammatory/immune responses," Proceedings of the National Academy of Sciences of the United States of America, vol. 105, no. 38, pp. 14638-14643, 2008.

[21] K. Németh, A. Leelahavanichkul, P. S. T. Yuen et al., "Bone marrow stromal cells attenuate sepsis via prostaglandin E 2dependent reprogramming of host macrophages to increase their interleukin-10 production," Nature Medicine, vol. 15, no. 1, pp. 42-49, 2009.

[22] J. H. Ylöstalo, T. J. Bartosh, K. Coble, and D. J. Prockop, "Human mesenchymal stem/stromal cells cultured as spheroids are selfactivated to produce prostaglandin E2 that directs stimulated macrophages into an anti-inflammatory phenotype," Stem Cells, vol. 30, no. 10, pp. 2283-2296, 2012.

[23] X.-X. Jiang, Y. Zhang, B. Liu et al., "Human mesenchymal stem cells inhibit differentiation and function of monocyte-derived dendritic cells," Blood, vol. 105, no. 10, pp. 4120-4126, 2005.

[24] A. J. Nauta, A. B. Kruisselbrink, E. Lurvink, R. Willemze, and W. E. Fibbe, "Mesenchymal stem cells inhibit generation and function of both CD34 +-derived and monocyte-derived dendritic cells," Journal of Immunology, vol. 177, no. 4, pp. 20802087, 2006.

[25] Y. P. Li, S. Paczesny, E. Lauret et al., "Human mesenchymal stem cells license adult CD $34^{+}$hemopoietic progenitor cells to differentiate into regulatory dendritic cells through activation of the Notch pathway," The Journal of Immunology, vol. 180, no. 3, pp. 1598-1608, 2008.

[26] S. Huber, N. Gagliani, E. Esplugues et al., "Th17 cells express interleukin-10 receptor and are controlled by Foxp3and Foxp3+ regulatory CD4+ T cells in an interleukin-10dependent manner," Immunity, vol. 34, no. 4, pp. 554-565, 2011.

[27] A. Chaudhry, R. M. Samstein, P. Treuting et al., "Interleukin10 signaling in regulatory $\mathrm{T}$ cells is required for suppression of Th17 cell-mediated inflammation," Immunity, vol. 34, no. 4, pp. 566-578, 2011.

[28] S. M. Anderton and S. Fillatreau, "Activated B cells in autoimmune diseases: the case for a regulatory role," Nature Clinical Practice Rheumatology, vol. 4, no. 12, pp. 657-666, 2008.

[29] K. Yanaba, J. Bouaziz, K. M. Haas, J. C. Poe, M. Fujimoto, and T. F. Tedder, "A regulatory $\mathrm{B}$ cell subset with a unique CD1dhiCD5+ phenotype controls T cell-dependent inflammatory responses," Immunity, vol. 28, no. 5, pp. 639-650, 2008.

[30] R. Madan, F. Demircik, S. Surianarayanan et al., "Nonredundant roles for B cell-derived IL-10 in immune counter-regulation," Journal of Immunology, vol. 183, no. 4, pp. 2312-2320, 2009. 
[31] E. Zappia, S. Casazza, E. Pedemonte et al., "Mesenchymal stem cells ameliorate experimental autoimmune encephalomyelitis inducing T-cell anergy," Blood, vol. 106, no. 5, pp. 1755-1761, 2005.

[32] R. Tasso, C. Ilengo, R. Quarto, R. Cancedda, R. R. Caspi, and G. Pennesi, "Mesenchymal stem cells induce functionally active T-regulatory lymphocytes in a paracrine fashion and ameliorate experimental autoimmune uveitis," Investigative Ophthalmology and Visual Science, vol. 53, no. 2, pp. 786-793, 2012.

[33] X. Zhang, X. Ren, G. Li et al., "Mesenchymal stem cells ameliorate experimental autoimmune uveoretinitis by comprehensive modulation of systemic autoimmunity," Investigative Ophthalmology \& Visual Science, vol. 52, no. 6, pp. 3143-3152, 2011.

[34] A. Peister, J. A. Mellad, B. L. Larson, B. M. Hall, L. F. Gibson, and D. J. Prockop, "Adult stem cells from bone marrow (MSCs) isolated from different strains of inbred mice vary in surface epitopes, rates of proliferation, and differentiation potential," Blood, vol. 103, no. 5, pp. 1662-1668, 2004.

[35] J. Tolar, A. J. Nauta, M. J. Osborn et al., "Sarcoma derived from cultured mesenchymal stem cells," Stem Cells, vol. 25, no. 2, pp. 371-379, 2007.

[36] D. J. Prockop and A. Keating, "Relearning the lessons of genomic stability of human cells during expansion in culture: implications for clinical research," Stem Cells, vol. 30, no. 6, pp. 1051-1052, 2012.

[37] G. Ren, J. Su, L. Zhang et al., "Species variation in the mechanisms of mesenchymal stem cell-mediated immunosuppression," Stem Cells, vol. 27, no. 8, pp. 1954-1962, 2009. 


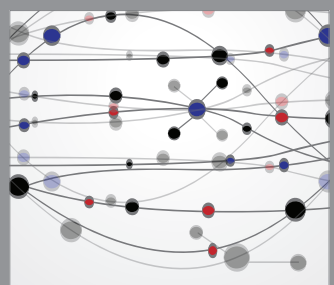

The Scientific World Journal
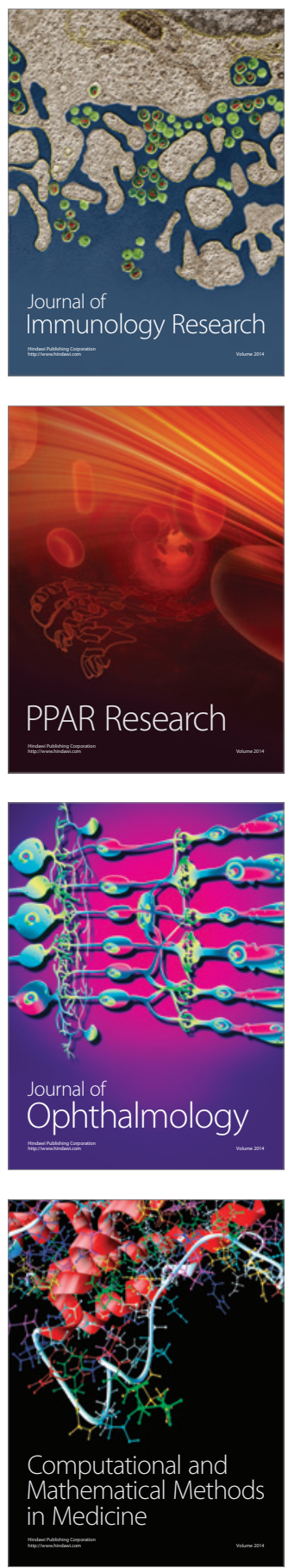

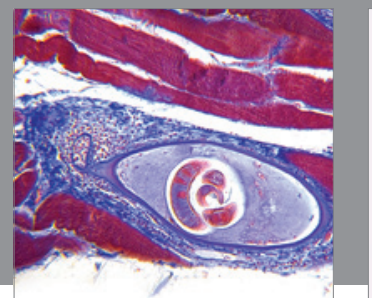

Gastroenterology

Research and Practice
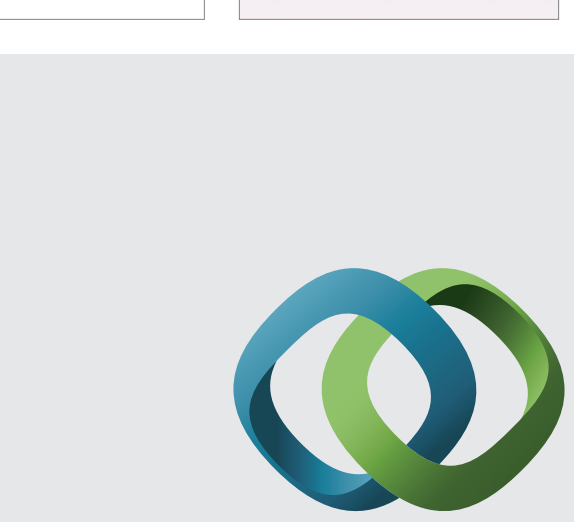

\section{Hindawi}

Submit your manuscripts at

http://www.hindawi.com
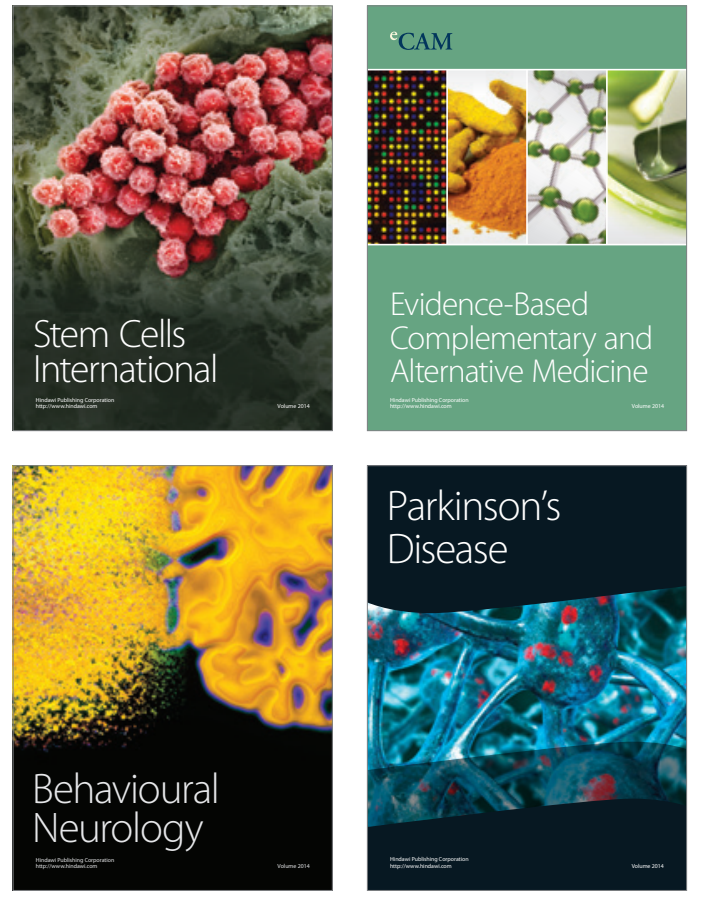
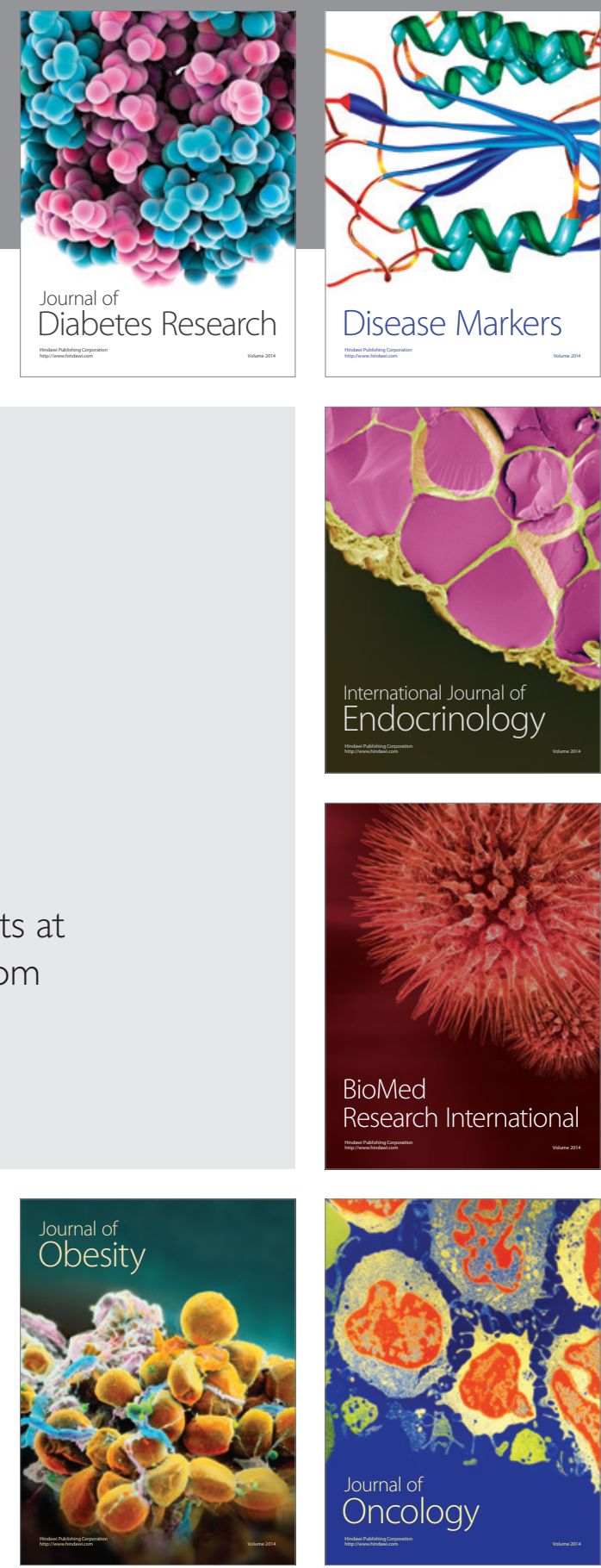

Disease Markers
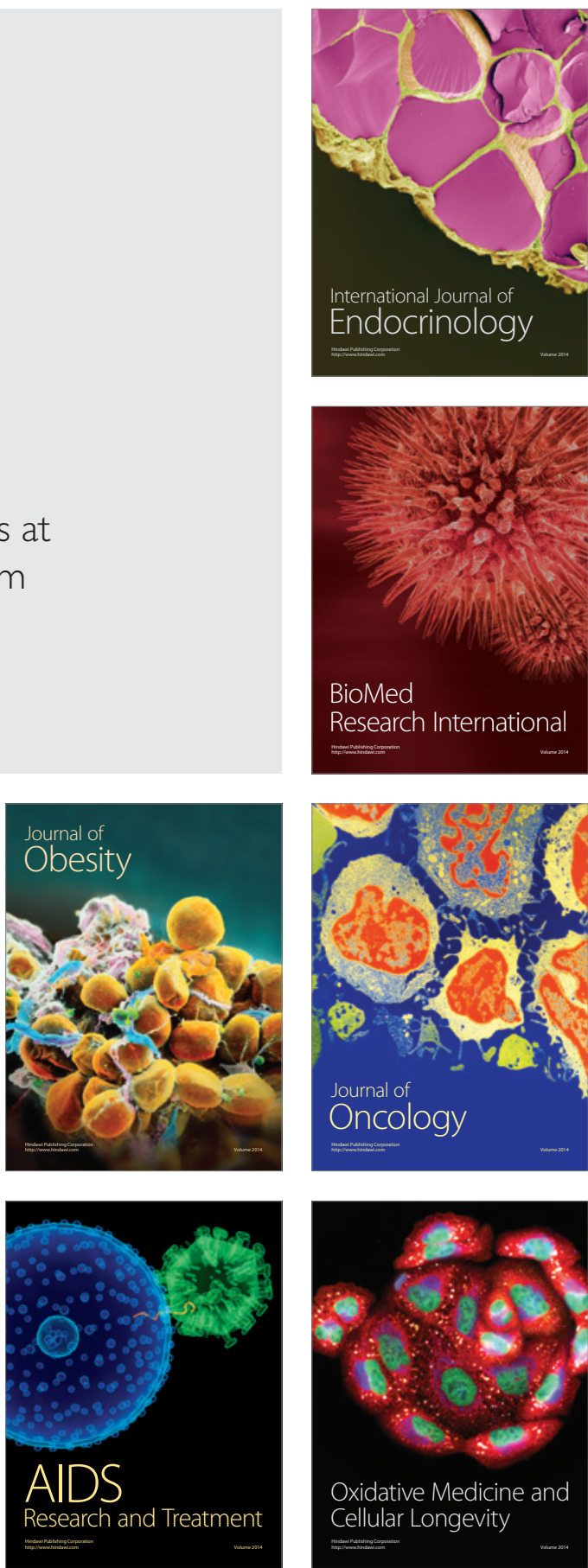